\title{
El método clínico, el método epidemiológico y la Epidemiología Clínica
}

\author{
The clinical method, the epidemiological method and the clinical \\ epidemiology
}

DraC. Silvia Martínez Calvo, MSc. Rina Milagros Ramis Andalia

Escuela Nacional de Salud Pública. La Habana, Cuba.

\begin{abstract}
RESUMEN
El objetivo de este trabajo es revisar críticamente la relación entre los métodos clínico y epidemiológico, su conjugación en la denominada Epidemiología Clínica y la posibilidad que brindan para la solución de los problemas de salud correspondientes. Se realizó una revisión documental sobre el tema y entre los resultados se destacan, la vigencia de ambos métodos para la investigación en salud y la aplicación de los procedimientos de la Epidemiología Clínica como disciplina de escaso desarrollo, especialmente cuando se refiere al ámbito nacional. Los métodos clínicos y epidemiológicos, no son divergentes, sino que se complementan para desarrollar un enfoque transdisciplinar, imprescindible para la investigación y la solución de los problemas de salud.
\end{abstract}

Palabras clave: método clínico, método epidemiológico, investigación, problema de salud.

\begin{abstract}
The objective of this paper is to review in a critical way the relationship between the clinical and the epidemiological methods, their conjugation in the so called clinical epidemiology and the possibilities they provide for the health problems resolution. A documentary review on this topic was made, and some of the significant results were the validity of both methods for health research and the implementation of the clinical epidemiology procedures, as a less developed discipline, particularly at domestic
\end{abstract}


level. It was concluded that both methods, rather than divergent, are supplementary to develop an indispensable transdisciplinary approach for the research and the resolution of health problems.

Key words: clinical method, epidemiological method, research, health problem.

\section{NTRODUCCI ÓN}

Se acepta desde sus orígenes, el aporte de la Clínica al desarrollo de la Epidemiología, conjuntamente con la Filosofía y la Estadística, después enriquecida con las propuestas de otras ciencias sociales. En tiempos recientes, parece olvidado este fundamento y se valoran los métodos clínico y epidemiológico, como totalmente divergentes y en ocasiones, productores de increíbles discrepancias. El posible origen de esta situación, tal vez se derive del objeto de estudio al que se aplican ambos métodos que, a pesar de los intentos de multidisciplinariedad en los inicios y transdisciplinariedad, mucho más reciente, mantienen esa bipolaridad "salud enfermedad" como divergentes y no como el proceso que definitivamente representan.

El fenómeno enfermedad contrapuesto al fenómeno salud, constituye el fundamento teórico del desempeño cotidiano, de una buena parte de los profesionales médicos clínicos. Desmenuzan la enfermedad en todos sus componentes así como en su proceso interno, con la aplicación del método clínico y sus procedimientos y consideran alcanzado su "enfoque integral", cuando entre otros elementos, incluyen los antecedentes patológicos personales, familiares y lo relativo a las etapas previas al cuadro clínico florido que muestra el paciente. Sin embargo, paradójicamente, los clínicos estudian más la enfermedad como fenómeno, que los epidemiólogos la salud como tal.

Para el profesional clínico las herramientas de abordaje de su objeto son harto conocidas, tienen reconocimiento científico histórico y actualmente alcanzan un nivel técnico elevado, que facilita su aproximación a la esencia. Quizás estos avances tecnológicos, hayan contribuido a subvalorar la aplicabilidad de los procedimientos convencionales y a "rutinizar" las destrezas y habilidades derivadas de su método particular. Por el contrario, para el epidemiólogo, el fenómeno salud se transforma en algo sumamente complejo, casi inabordable a partir de la carencia de instrumentación y procedimientos, de ahí que prefiera la denominación del proceso salud enfermedad, que le facilita compartir ambos fenómenos, muchas veces con la utilización de los instrumentos de disciplinas clínicas y sociales, incorporadas con cierta inhabilidad a su arsenal profesional.

Ante los actuales perfiles de salud y enfermedad de la población cubana, resulta pertinente revisar algunos vínculos entre el método clínico y el método epidemiológico, que constituyen los métodos conductores para los procesos de revitalización o renovación del sistema de salud, para contribuir a los cambios que se requieren. En ese sentido, el objetivo de este trabajo es revisar críticamente la relación entre ambos métodos, su conjugación en la denominada Epidemiología Clínica y la posibilidad que brindan para la solución de los problemas de salud correspondientes. 


\section{MÉTODOS}

Se realizó una revisión documental sobre el tema y se compararon los resultados con lo que ocurre en el ámbito nacional en cuanto a la relación entre el método clínico y el método epidemiológico, así como el desarrollo y aplicación de la Epidemiología Clínica.

\section{Método clínico y método epidemiológico}

La comparación entre ambos métodos, ha sido un ejercicio simple de obligatoria aparición en los textos de Epidemiología que fueron elaborados en las décadas de los 60 y los 70 del pasado siglo. Resulta clásico, un cuadro que enumera los pasos para desarrollar cada método por separado, en una forzada y forzosa comparación que, a nuestro juicio, contribuyó a dicotomizar un objeto de estudio que definitivamente pugnaba por su análisis integral.

Aquel famoso parangón en que el método clínico desarrollaba el "diagnóstico clínico" y el método epidemiológico descansaba en la "hipótesis epidemiológica", se afianzó en el quehacer de los profesionales de tal forma, que se perdió la noción del proceso salud enfermedad, observado desde visiones particulares, pero totalmente conjugadas o integradas. Se describían otros elementos "diferenciables" que incluían hasta los modelos para plasmar o consignar los hallazgos clínicos y epidemiológicos y así se comparaba la Historia Clínica con la Ficha o Informe Epidemiológico, los medios auxiliares de diagnóstico, entre otros. ${ }^{1}$

En décadas posteriores al propio siglo xx y ya en el siglo xxI, se perfecciona y amplía el objeto de estudio de la Epidemiología, siempre derivado de las consecuencias que los riesgos, enfermedades y daños ocasionan en las personas, ya sea individual o colectivamente. No se perdió el enfoque integral del proceso salud enfermedad, aunque la tendencia a estudiar la enfermedad, prevaleció ante el estudio de la salud. Se recurre al enfoque integral como si con esa simple mención, inmediatamente pudiera lograrse su desarrollo y se utilizan los términos multidisciplinariedad e interdisciplinariedad, como representaciones erróneas de esa integralidad de disciplinas y métodos. En ese sentido, surge un proceso renovador, sustentado en la transdisciplinariedad, reconocida como una interacción disciplinar según la propuesta de Nicolescu que conjuntamente con Edgar Morin, representan dos de los más importantes ideólogos del pensamiento transdiciplinar. ${ }^{2,3}$

Con la visión transdisciplinar, se reconoce la realidad multidimensional y estructurada en múltiples niveles, que remplaza la realidad unidimensional de un solo nivel en el pensamiento clásico. Es complementaria al enfoque disciplinario; de la confrontación de las disciplinas hace surgir nueva información, que articula entre si y ofrece una nueva visión sobre la naturaleza y la realidad. No busca el dominio de varias disciplinas, sino la apertura a aquellas que las atraviesan y las trascienden. ${ }^{2,4}$

Esta propuesta, pudiera ajustarse perfectamente a esa relación que ha existido entre la Clínica y la Epidemiología y posiblemente, entre la Epidemiología y el resto de las disciplinas, pilares de su formación; pero este nivel de interacción se ha limitado a la semántica y a la transferencia de conocimientos, mientras que el resto de los niveles en realidad han funcionado de forma separada. Existen pues, nuevas formas de interacción entre estas dos disciplinas, que pueden aprovecharse para rebasar las limitaciones impuestas por el pensamiento estrictamente disciplinar.

Para desarrollar ese vínculo, ambas disciplinas debían avanzar simultáneamente. ¿Qué ocurrió con las Ciencias Clínicas? Después de la Segunda Guerra Mundial, la Clínica se desarrolla en los hospitales, conjuntamente con la Microbiología, el 
Laboratorio Clínico y el de Rayos X y la Anatomía patológica. El individuo enfermo se mantiene como su objeto de estudio y los descubrimientos científicos en etapas posteriores, elevan el desempeño de los especialistas clínicos, aunque ese avance se sustenta, básicamente, en la aplicación de la tecnología, no para precisar el diagnóstico clínico, sino más bien, con la enorme tendencia de sustituir esa clásica habilidad diagnóstica, por un criterio eminentemente derivado de los resultados que ofrecen los equipos de última generación.

¿Qué ocurrió con la Epidemiología? El método epidemiológico, en los servicios, estuvo enfocado al control de los programas y las enfermedades infecciosas y en la dimensión investigativa, la aplicación de una Epidemiología por excelencia cuantitativista, que se inició con la denominada transición epidemiológica de las enfermedades infecciosas a las no infecciosas, con su máxima expresión en la Epidemiología de los factores de riesgo, no siempre bien utilizada.

Regresa la Epidemiología a sus orígenes con la aplicación de la Estadística, ahora como tecnología instrumental privilegiada, que relega el uso de los enfoques cualitativos para la identificación de los factores causales que tanto influyen en los problemas de salud de la población. Han sido bien criticados estos enfoques exageradamente "cuantitativistas" de la Epidemiología e incluso, se sugiere modificarlos ante los retos del actual siglo. ${ }^{5}$ Se complica más esa instrumentación tecnológica, al pretender conjugar el método clínico y el método epidemiológico, mediante lo que algunos consideran una nueva disciplina: la Epidemiología Clínica.

\section{¿Es la Epidemiología clínica, otra Epidemiología?}

Cuando la Epidemiología emerge como Ciencia en el siglo xIX, ya era una práctica muy antigua. Su origen más documentado se remonta a la antigua Grecia y posteriormente, cuando en el mundo científico denominémosle así- se contendía entre la teoría miasmática y la teoría contagionista y aún se desconocían las causas de las enfermedades, destacaron precursores capaces de describir sus mecanismos de producción. Son antológicos los estudios de James Lind sobre la naturaleza y la causa del escorbuto, publicados en 1753, o el ensayo sobre la causa del cólico epidémico de Devonshire en 1767. Marca un hito en la historia de la Epidemiología, el estudio y control del brote epidémico de Cólera realizado en Broad Street, Londres, 1854, que consagra a John Snow, como el "Padre de la Epidemiología". Situación similar enfrentaría años después en la Habana, el brillante medico oftalmólogo, Carlos J Finlay, descubridor del mosquito agente transmisor de la fiebre amarilla, ante un brote de cólera en la barriada del Cerro (1876). A finales del siglo xIX, se consolida el vínculo clínico epidemiológico, con el descubrimiento por Pasteur de los agentes causales de las enfermedades infecciosas. ${ }^{6,7}$

Este largo y fructífero camino de experiencias e interrelaciones entre ambas disciplinas, facilitaron a J ohn R. Paul la publicación en 1958 del primer libro reconocido sobre Epidemiología Clínica. Su visión de esta disciplina se orientaba a los estudios de caso, sin involucrarse en las incidencias poblacionales, aunque reconocía que, tanto la Epidemiología Clínica como la no clínica y sus principios, pueden formar parte del trabajo del médico. ${ }^{8}$ Así se pretendía fortalecer esa interrelación disciplinar, aunque con la incorporación de otra disciplina: la Bioestadística.

Se abrió el espacio para que varios eminentes clínicos, aportaran nuevos elementos para el desarrollo de la Epidemiología Clínica, como Alvan Feinstein, con una importante obra inicial dedicada a la experimentación clínica, aspecto hoy devenido en paradigma de la investigación científica por la denominada Medicina Basada en la 
Evidencia. ${ }^{9}$ Ineludible la mención a David L. Sackett, uno de los más importantes continuadores de esta ideología científica, en cuya obra inicial puntualiza que:

La aplicación de los principios epidemiológicos y unos cuantos más de la bioestadística a las creencias, juicios e intuiciones que comprenden el arte de la medicina, podían mejorar sustancialmente la exactitud y la eficacia del diagnóstico, del pronóstico, del tratamiento, el mantenerse al día, y de importancia especial, la capacidad de enseñar a los demás cómo hacer estas cosas. ${ }^{10}$

Así surge, en nuestra opinión, la actual Epidemiología Clínica, como expresión de una tendencia extrema del positivismo, con indiscutibles ventajas en el ámbito de la investigación clínico epidemiológica, aunque extremadamente reduccionista, con implicaciones científicas e ideológicas en el desarrollo de ambas disciplinas y en la Salud Pública. Entre sus elementos cuestionables se consideran:

- encomiar la medición como expresión de seguridad y certeza científica,

- promoverse como ideología científica del pensamiento neoliberal en función del desarrollo, la eficiencia y la eficacia en los servicios clínicos, con menoscabo de la Salud Pública y los presupuestos de la Medicina Social.

Las bases científicas de las disciplinas que conforman la Epidemiología Clínica, deben ajustarse a las corrientes epistemológicas vigentes, si se pretende avanzar en un verdadero enfoque integral de sus métodos. Como expresa Capra:

El origen de nuestro dilema reside en nuestra tendencia a crear abstracciones de objetos separados, incluyendo un sí mismo independiente, para creer después que pertenecen a una realidad objetiva, dotada de existencia autárquica (autosuficiente). Para superar esta ansiedad cartesiana, necesitamos pensar sistémicamente, desplazando nuestra atención conceptual de los objetos a las relaciones. Sólo entonces podremos comprender que identidad, individualidad y autonomía no significan separatividad e independencia. Para recuperar nuestra plena humanidad, debemos reconquistar nuestra experiencia de conectividad con la trama entera de la vida. ${ }^{11}$

Estas valoraciones, para nuestro contexto, propiciaría no situarse en posiciones extremas, no esperar de la Epidemiología Clínica y de la Medicina Basada en la Evidencia, resultados más allá de sus potencialidades; ajustarlas al momento y lugar necesarios y sobre todo, asumir con cautela que el experimento científico, es el "estándar de oro" de la investigación científica. ${ }^{12}$

\section{Desarrollo de la Epidemiología Clínica en Cuba}

Son reconocidos los vínculos entre ambas disciplinas desde los inicios de la Medicina y la Salud Pública en Cuba, con ejemplos puntuales, tal como se describió en párrafos anteriores. La práctica de la medicina privada, durante la etapa republicana, no constituyó espacio idóneo para la aplicación e interrelación del método clínico y el método epidemiológico y aún estaban lejanas las propuestas de la Epidemiología Clínica. Con la creación en la década del 60, del Sistema Nacional de Salud, al establecerse las bases teóricas y metodologías de la docencia, la investigación y los servicios en Epidemiología, se pretendió impulsar - no siempre con éxito- el vínculo 
entre la Clínica y la Epidemiología en todos los niveles de atención del Sistema Nacional de Salud, especialmente en el nivel primario de atención.

No fue hasta la década del 90, que se inició paulatinamente la introducción de los conceptos, métodos y procedimientos de la Epidemiología Clínica en el área hospitalaria, con énfasis en los enfoques preconizados por Alvan Feinstein y David Sackett y debidamente adaptados a nuestros principios científicos.

En 1992, la figura del clínico epidemiólogo, como jefe de departamento de Epidemiología en los hospitales, no tuvo la aceptación esperada en los centros seleccionados en la capital del país, al considerarse erróneamente, que la práctica clínica se reduciría a la aplicación de guías, algoritmos y decisiones matemáticas, aspectos poco compatibles con el tradicional método clínico establecido en las facultades de medicina cubanas.

El resultado de la no aceptación en las unidades de servicios, fue el avance de la Epidemiología Clínica en los centros nacionales de investigación científica, exclusivamente en el área de aplicación de ensayos clínicos y validación de pruebas diagnosticas, no así en la práctica clínica y epidemiológica. Las competencias de los epidemiólogos cubanos, priorizan las acciones de vigilancia, promoción y control y la investigación clínico epidemiológica, aún tiene escaso desarrollo. Se requieren cambios en el enfoque y manejo actual de este tema, si deseamos que se reconozca el vínculo de la Clínica y la Epidemiología en un nuevo nivel y se desarrolle como exigen los actuales perfiles de morbilidad y mortalidad en Cuba.

\section{CONSI DERACI ONES FI NALES}

La revitalización del método clínico, como parte de las transformaciones necesarias del Sistema Nacional de Salud cubano, requiere de incorporar el pensamiento epidemiológico al desempeño de los profesionales de las Ciencias Clínicas en un nivel de desarrollo muy superior al actual, para convertir en acciones un discurso en ocasiones obsoleto, si se compara con los avances que mundialmente poseen la Clínica y la Epidemiología.

Si se modifican los objetos en ambas disciplinas, ineludiblemente se modifican o ajustan sus métodos y la demora en aceptar esas modificaciones o ajustes, tendrá consecuencias para la atención de salud a corto, mediano y largo plazo.

\section{REFERENCI AS BI BLI OGRÁFICAS}

1. Armijo R. Epidemiología. T I. 2da ed. Epidemiología Básica. Buenos Aires: Intermédica Editorial; 1978.

2. Nicolescu B. La Transdisciplinariedad, Manifiesto. Sonora: 7 Saberes; 1996.

3. Morin E. Los siete saberes necesarios en la educación del futuro. Lima: Editora Magisteria; 2007.

4. Almeida N. Complejidad y transdisciplinariedad en el campo de la salud colectiva: evaluación de conceptos y aplicaciones. Salud Colectiva. 2006;2:2. 
5. Kaplan J, Thacker S, Lezin N. Epidemiology in the $21^{\text {st }}$ Century: Comunication, Calculation, and Intervention. Amer J Public Health. 1999; 89(8):1153-5.

6. Castillo M. Epidemiología. La Habana: Editorial Pueblo y Educación; 1984.

7. Back C, Llopis A, Najera E, Terris M. El Desafío de la Epidemiología. Washington: OPS; 1988. (Publicación científica No. 505).

8. Paul RJ . Clinical Epidemiology. Chicago: The University Chicago Press; 1958.

9. Feinstein AR. Clinical Biestatistics. Missouri: The CV Mosby Company; 1977.

10. Sackett D, Haynes B, Tugwell P. Epidemiología clínica: una ciencia básica para la medicina clínica. Madrid: Díaz de Santos; 1989.

11. Capra F. La trama de la vida. 2da ed. Barcelona: Anagrama S.A.; 1999.

12. Ramis Andalia R. Origen e intencionalidad de la Epidemiología clínica. Rev Cubana Salud Pública [Internet]. 2005 [citado 10 J un 2012 ]; 31(3). Disponible en: http://scielo.sld.cu/scielo.php?script=sci arttext\&pid=S0864 34662005000300009\&lng=es

Recibido: 25 de marzo de 2012.

Aprobado: 25 de abril de 2012.

Silvia Martínez Calvo. Escuela Nacional de Salud Pública. Calle 100 No. 1132 e/ E y Perla. Altahabana, municipio Boyeros. La Habana, Cuba. 